\title{
Adults' strategy choices for simple addition: Effects of retrieval interference
}

\author{
JAMIE I. D. CAMPBELL and JENNIFER C. TIMM \\ University of Saskatchewan, Saskatoon, Saskatchewan, Canada
}

\begin{abstract}
Simple addition (e.g., $3+2,7+9$ ) may be performed by direct memory retrieval or by such procedures as counting or transformation. The distribution of associations (DOA) model of strategy choice (Siegler, 1988) predicts that procedure use should increase as retrieval interference increases. To test this, 100 undergraduates performed simple addition problems, either after blocks of simple multiplication (high-interference context) or after blocks of simple division problems (low-interference context). Addition took longer and was more error prone after multiplication; in particular, there were more multiplication confusion errors on the relatively easy, small-number addition problems (e.g., $3+$ $2=6,4+3=12$ ), but not on the more difficult, large-number additions. Consistent with the DOA, participants reported greater use of procedures for addition after multiplication, but more so for small addition problems. The findings demonstrate that adults' use of procedural strategies for simple addition is substantially influenced by retrieval interference.
\end{abstract}

The distinction between declarative and procedural knowledge is a fundamental dichotomy with respect to human memory and is central to our understanding of automatic processing and skill acquisition (J. R. Anderson \& Lebiere, 1998; Logan, 1988). Calculation skills have provided an important domain for the study of the theoretical relation between declarative and procedural memory (Logan \& Klapp, 1991; Sohn \& Carlson, 1998; Zbrodoff, 1995); in addition, the use of declarative fact retrieval versus procedural strategies for calculation is also of substantial practical importance. For example, recent studies comparing simple arithmetic performance of East Asian and North American university students show that cross-national differences favoring East Asian populations are due, in part, to North American adults' lower reliance on direct memory retrieval for simple arithmetic (e.g., $6+8=14,7 \times 9=63$ ) and relatively greater reliance on less efficient procedural strategies, such as counting or transformation (e.g., $6+7=6+6+1$; Geary, 1996; LeFevre \& Liu, 1997). In fact, North American university students do not report exclusive reliance on retrieval for simple addition (Geary, 1996; Geary \& Wiley, 1991; LeFevre, Sadesky, \& Bisanz, 1996), multiplication (LeFevre, Bisanz, et al., 1996), division (LeFevre \& Morris, 1999), or subtraction (Geary, Frensch, \& Wiley, 1993). Thus, understanding the cognitive mechanisms that mediate arithmetic strategy choice contributes to understanding individual and cross-national differences in mathemat-

\footnotetext{
This research was funded by a grant from the Natural Sciences and Engineering Research Council of Canada. The authors thank John T. Wixted, Mark Ashcraft, and an anonymous reviewer for helpful comments on a previous version of this manuscript. Correspondence concerning this article should be addressed to J.I.D. Campbell, Department of Psychology, University of Saskatchewan, 9 Campus Drive, Saskatoon SK, S7N 5A5 Canada (e-mail: jamie.campbell@usask.ca).
}

ical competence, as well as to understanding the relation between declarative and procedural memory processes. In the following experiment, we investigated the possible role of retrieval interference in adults' use of direct retrieval versus procedural strategies for simple addition.

A prominent theory of strategy choice in cognitive arithmetic is Siegler's adaptive strategy choice model (ASCM; Siegler \& Lemaire, 1997; Siegler \& Shipley, 1995). The ASCM and its less comprehensive predecessor, the distribution of associations (DOA) model (Siegler, 1988; Siegler \& Shrager, 1984), have been successfully applied to explain strategy choice and performance in a wide variety of cognitive skills. The relatively simple DOA model, which was developed to explain children's arithmetic strategy choices, may provide an appropriate model for adults. According to the DOA model, direct retrieval is the default strategy for simple arithmetic problems. Procedural strategies are employed only when retrieval attempts do not yield an answer with sufficient memory strength. Specifically, individuals set a minimum confidence criterion and a maximum search length for retrieval. The confidence criterion specifies the minimum strength required for an answer to be produced. Search length determines the maximum elapsed time before the retrieval attempt is abandoned. If no candidate answer exceeds the confidence criterion within the deadline for retrieval, an alternative strategy is employed. Thus, predictions from the DOA model regarding strategy choice are directly linked to assumptions about number fact retrieval.

Like most current models of arithmetic memory, the DOA model assumes that number fact retrieval involves parallel activation of a network of related arithmetic facts in long-term memory. When a problem is presented (e.g., $3+4=$ ?), the correct fact $(3+4=7)$ is activated, as well as other facts associated with the problem's operands (e.g., $3+5=8,2+4=6,3 \times 4=12$ ). Error analyses sug- 
gest that the most strongly activated facts are those that share features with, and are numerically close to, the presented problem (Ashcraft, 1992; Campbell, 1995; Siegler, 1988). Encoding a problem causes activation to be distributed among related facts, and the retrieval probability for each fact is determined by its relative strength. Thus, if associative competitors are strongly activated, the relative activation of the correct fact is lower, and performance will be characterized by slower, more error-prone retrieval. ${ }^{1}$

In overview, the DOA model assumes that (1) the efficiency of number fact retrieval is determined by the relative strength of the correct fact in the context of associative competitors activated by the problem, (2) no activated answer is produced unless its relative strength exceeds the confidence criterion for retrieval, and (3) if no answer exceeds criterion within the search length for retrieval, an alternative strategy, such as counting or transformation, is employed. Consequently, changing a problem's distribution of activation among associated facts should influence use of direct retrieval versus nonretrieval strategies. Specifically, practicing a problem (e.g., solving $3 \times 4=$ ?) should strengthen this fact in memory (i.e., $3 \times 4=12$ ) and increase the speed and probability of a correct retrieval in subsequent tests of that problem. Increasing the strength of one fact, however, should also interfere with retrieval of related facts. For example, practicing $3 \times 4=12$ ought to interfere with retrieval of $3+$ $4=7$, because strengthening this competing fact would reduce the relative strength of the correct association for $3+4$. As a result, retrieval should be slower and more error prone. Furthermore, according to the DOA model, increasing retrieval interference by strengthening associative competitors should also decrease use of retrieval strategies and increase use of procedural strategies. This is because sufficiently strong activation of one or more competitors may result temporarily in a distribution of activation in which no fact, including the correct association, exceeds the relative strength criterion for retrieval.

To test this prediction, we created high- and lowinterference contexts for simple addition by first giving participants several blocks of simple multiplication (M-A group) or several blocks of simple division (D-A group), respectively. Several types of evidence indicate that addition performance is susccptible to interference from multiplication (see Miller \& Paredes, 1990). For example, in adults' true-false arithmetic verification, confusion products (e.g., $2+4=8,3+4=12$ ) produce marked slowing and increased errors, relative to controls (e.g., $2+4=7,3+4=11$; Zbrodoff \& Logan, 1986). Furthermore, stating the product instead of the sum is a common type of addition error when both multiplication and addition are tested under speed pressure (Campbell, 1994). In contrast, we do not expect such interference of addition performance in connection with division. This is because simple multiplication and addition share basic features that simple division and addition do not. Both addition and multiplication are commutative operations (i.e., operand order is irrelevant); indeed, transfer studies indicate that commuted pairs (e.g., $3 \times 6$ and $6 \times 3$ ) access a common memory node (Rickard \& Bourne, 1996). This cannot be true for division (i.e., $6 \div 3 \neq 3 \div 6$ ). Furthermore, the two operands for simple addition and multiplication problems are always single-digit numbers (e.g., $8+2,8 \times 2$ ), whereas for the majority of simple division problems, the left operand (i.e., the dividend) is a two-digit number (e.g., $16 \div 2$ ). The greater conceptual and structural similarity of simple multiplication and addition problems make it more likely that multiplication practice will interfere with addition than that division practice will. Thus, we expected correct response times (RTs), error rates, and the reported use of nonretrieval strategies for addition to be greater overall after multiplication than after division.

Moreover, we expected effects of interference, including increased use of procedural strategies, to be stronger for the easier, small-number addition problems. This is a counterintuitive prediction. Both children and adults report using nonretrieval procedures more for numerically large problems $(7+6)$ than for small problems $(2+3$; Geary, 1996; LeFevre, Sadesky, \& Bisanz, 1996; Siegler, 1988). Small-number problems are encountered more frequently (Ashcraft \& Christy, 1995) and therefore are more likely to have high relative strength of the correct association. Better memorization of small-number facts, together with less use of nonretrieval strategies, explains the common finding that performance is generally better for small- than for large-number facts (Geary, 1996). Despite the fact that small-number problems are generally easier than large-number problems, we nonetheless expected the multiplication context to interfere more with performance of smaller additions. Campbell (1994) found that most multiplication confusion errors in addition (e.g., $2+3=6,3+5=15$ ) occur on the small-number addition problems with both addends less than or equal to 5 . This may occur because small multiplication facts have greater memory strength than larger multiplication problems; consequently, small-number products $(2 \times 3=6)$ would be activated more easily by the corresponding addition operands $(2+3=$ ?). The greater susceptibility of small addition to multiplication confusions suggests that interference from prior multiplication ought to be especially strong for smaller addition problems.

In the present experiment, participants identified their strategy after answering each arithmetic problem. Selfreported strategies for simple arithmetic may be susceptible to instructional biases (Kirk \& Ashcraft, 1997), but previous research in which adults' strategy reports for simple arithmetic were examined suggests that they are generally valid (e.g., Geary et al., 1993; LeFevre, Bisanz, et al., 1996; LeFevre, Sadesky, \& Bisanz, 1996). Furthermore, our experiment tested specific predictions for relative differences in strategy reports as a function of interference and problem size that are not troubled by instructional bias concerns. 


\section{METHOD}

\section{Participants}

One hundred volunteers ( 67 females and 33 males) participated to fulfill a research option in their introductory psychology course. The experiment was described as a test of simple arithmetic skills on the participant sign-up sheet. Ages ranged from 17 to 38 years, with a mean age of 19.2 years.

\section{General Procedure and Apparatus}

Testing occurred in a quiet room with an experimenter present and required about $35 \mathrm{~min}$. The experimenter explained that several tasks would be administered: a block of 20 number-naming trials, 4 blocks of 36 multiplication or division trials, followed by 2 blocks of 36 addition trials. The participants were offered a brief break between blocks. Stimuli were presented on two high-resolution monitors, using an IBM-type personal computer, with one monitor viewed by the experimenter and the other viewed by the participant. Stimuli consisted of arabic digits $7 \mathrm{~mm}$ high $\times 3 \mathrm{~mm}$ wide and appeared as white characters against a dark background. The participants wore a lapel microphone connected to a sound-activated relay that controlled a software clock accurate to $\pm 1 \mathrm{msec}$. Prior to arithmetic trials, the participants performed a 20-trial speeded-naming task involving one- or two-digit numbers. This allowed the participants to find a comfortable viewing distance, accustomed them to rapid responding, and permitted adjustments to the sensitivity of the voice-activated relay.

\section{Arithmetic Task}

Stimuli. Addition and multiplication problems involved all pairs of single-digit numbers between 2 and 9 (i.e., $2+2$ to $9+9$ and $2 \times 2$ to $9 \times 9$ ). There are 36 possible pairings of the numbers 2 through 9 when commuted pairs (e.g., $4+5$ and $5+4)$ are counted as one problem. This includes 8 ties (e.g., $2+2,3+3$ ) and 28 nonties (e.g., $3+8,5+7)$. Division problems corresponded to the 36 multiplication problems (i.e., $6 \times 9$ corresponds to $54 \div 6$ or $54 \div 9$ ). The problems were presented horizontally, with the operands separated by the operation sign flanked by blank spaces.

Small addition and multiplication problems were defined as those with both operands less than or equal to 5 , whereas large problems had at least one operand greater than 5 (see Campbell, 1994; Noël, Fias, \& Brysbaert, 1997). Addition problems with both operands $\leq 5$ account for the vast majority of operation errors $(2+$ $3=6$; Campbell, 1994). If such errors index susceptibility to interference from multiplication, defining the small and large problem sets accordingly ought to maximize the chances to detect the predicted group $X$ size interactions. Division problems were classified as small if both the divisor and the quotient were less than or equal to 5 . Given these criteria, there are 10 small and 26 large problems in each operation.

Design. The participants received six blocks of 36 trials. Half of the participants received multiplication in Blocks 1-4 and addition in Blocks 5-6 (M-A group), and the other half received division in Blocks $1-4$ and addition in Blocks 5-6 (D-A group). The participants were assigned to the $M-A$ or $D-A$ condition on the basis of the order in which they entered the experiment (i.e., their participant number). Half of the $M-A$ and $D-A$ participants were instructed to provide a strategy report on each trial during the first four blocks. All the participants reported their strategy for each addition trial. For a given operation, all 36 problems were tested in random order within each block. Operand order for nontie problems (e.g., $6+9$ vs. $9+6,54 \div 6$ vs. $54 \div 9$ ) was determined randomly in the first block and then alternated across blocks.

Procedure. The participants were asked to respond accurately and quickly but were told that occasional errors are normal. The ex- perimenter pressed a key to initiate each block, and a message displayed at the beginning of each block indicated which operation would be tested. Prior to the first block of trials requiring strategy reports, the following instructions appeared on the monitor and were read out loud by the experimenter: "After each problem please indicate how you solved the problem by choosing from among the following strategies: Transform, Count, Remember, Other. Say TRANSFORM if you used knowledge of a related problem. Say COUNT if you used a strategy based on counting. Say REMEMBER if the answer seemed to come to you without any intermediate steps, inferences, or calculations. Choose OTHER if you used some other strategy or are uncertain." For reference during arithmetic trials, the participants also received a sheet of strategy descriptions as follows.

Transform: You solve the problem by referring to a related problem in the same or another operation. For example, you might solve $17-9=$ ? by remembering that $17-10=7$ so $17-9$ must equal 8 .

Count: You solve the problem by counting a certain number of times to get the answer.

Remember: You solve the problem by just remembering or knowing the answer directly from memory without any intervening steps.

Other: You may solve the problem by a strategy unlisted here, or you may be uncertain how you solved the problem.

Prior to each arithmetic trial, a fixation dot appeared and flashed twice over a $1-\mathrm{sec}$ interval at the center of the screen. The problem appeared (synchronized with the monitor's raster scan) on what would have been the third flash with the operation $\operatorname{sign}(x, \div,+)$ at the fixation point. Timing began when the problem appeared and ended when the sound-activated relay was triggered. Triggering the relay caused the problem to disappear immediately. This allowed the experimenter to mark RTs as spoiled when the microphone failed to detect the onset of the response. Immediately after the response, the prompt "Strategy Choice" appeared at the center of the screen with the words "Transform, Count, Remember, Other" centered immediately below. The four words were separated by six spaces and always appeared in the same order. The experimenter recorded the strategy reported by pressing one of four buttons on the computer keyboard. Once the strategy was recorded and the experimenter had entered the stated arithmetic answer, the screen cleared and displayed the fixation dot for the next trial. No feedback about speed or accuracy was provided during the experiment.

Immediately after the addition trials, the participants were asked to rate confidence in their addition strategy reports on a 5-point scale. For the 100 participants, the distribution of responses was very low, 0 ; low, 9; moderate, 35 ; high, 43; and very high, 13 . Thus, the majority rated their confidence as high or very high, and fewer than $10 \%$ reported low confidence. Both the $\mathrm{M}-\mathrm{A}$ group and the D-A group had mean confidence ratings of 3.6 .

\section{RESULTS $^{2}$}

A total of 1,736 RTs $(8.0 \%)$ were flagged for not coinciding exactly with the onset of the response. Another 519 RTs $(2.4 \%)$ were discarded as outliers more than $2.5 S D$ s from each participant's grand mean for each operation $\times$ size cell. Analyses of varaince (ANOVAs) of mean RT, percentage of error, and percentage of retrieval reported are presented separately for the context and the addition trials. For simplicity, we refer to retrieval rather than to reported retrieval. For the analyses of retrieval use, it made no difference whether or not error trials were included; consequently, these analyses were based on all the trials. 
Table 1

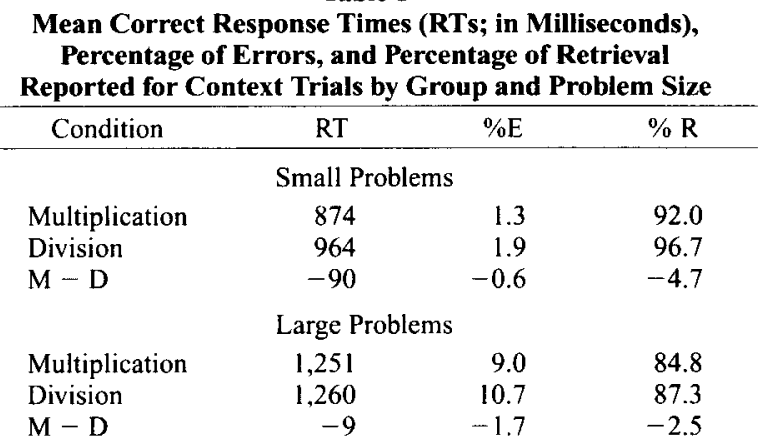

Note-Small, both multipliers or both the divisor and the quotient $\leq 5$ $(n=40)$; large, at least one factor $>5(n=104)$.

\section{Context Trials: Division and Multiplication Performance}

Table 1 includes the mean RT for correct trials, the mean percentage of errors, and the mean percentage of retrieval (i.e., selection of remember from our strategy list) for the context trials as a function of group (i.e., division or multiplication) and problem size (i.e., small or large). See Table 2 for the corresponding ANOVAs.

Response times. As is typically found, the participants took longer to answer large problems $(1,256 \mathrm{msec})$ than small problems $(919 \mathrm{msec})$. Mean RT was similar for multiplication $(1,063 \mathrm{msec})$ and division $(1,112 \mathrm{msec})$, however, and the effect of problem size on RT (i.e., mean RT for large problems - mean RT for small problems) did not differ significantly between multiplication $(+377 \mathrm{msec})$ and division $(+296 \mathrm{msec})$.

Errors. The participants produced more errors on large problems $(9.8 \%)$ than on small problems $(1.6 \%)$, illustrating typical effects of problem size. Error rates did not differ between multiplication (5.2\%) and division $(6.2 \%)$, and the effect of problem size on errors was equivalent for multiplication $(+7.7 \%)$ and division $(+8.8 \%)$.

Reported use of retrieval. Strategy reports for context trials were collected from $25 \mathrm{M}-\mathrm{A}$ and $25 \mathrm{D}-\mathrm{A}$ participants. Mean rates of retrieval, transformation, and counting were $86.8 \%, 3.3 \%$, and $8.9 \%$, respectively for multiplication and $89.9 \%, 3.0 \%$, and $5.2 \%$ for division. The participants selected the other category on fewer than $2 \%$ of the context trials. Retrieval use did not differ between multiplication and division, but as in previous research, the participants reported using retrieval more for small problems $(94.4 \%)$ than for large problems $(86.1 \%)$. The effect of problem size on retrieval did not differ between multiplication $(-7.2 \%)$ and division $(-9.4 \%)$.

Summary. The multiplication and division context groups presented similar performance in terms of RT, errors, and percentage of retrieval reported. These results may be somewhat surprising, given previous experiments showing better performance for multiplication than for division (Campbell, 1997, 1999; LeFevre \& Morris, 1999; Rickard \& Bourne, 1996). ${ }^{3}$ Percentage of retrieval for multiplication here $(87 \%$ ) was similar to LeFevre, Bisanz, et al. (1996; about 85\%), but LeFevre and Morris (1999) found much less retrieval in undergraduates' self-generated strategy descriptions for division and multiplication $(<60 \%)$. Perhaps our rather generic transformation category was not well understood in relation to division and multiplication, although the participants rarely selected the other category, which was to be used when uncertain. Our large samples $(n s=50)$ ought to provide reliable results, however, and we do not know whether to attribute the differences to the underlying populations or to procedural differences, although the latter seem more likely. One potentially important procedural difference was that our experiment compared multiplication and division between subjects, whereas operation typically is manipulated within subjects. As the following analyses demonstrate, the context in which an arithmetic operation is tested can have strong effects on performance and strategy reports.

\section{Addition Trials}

Table 3 presents mean RT for correct trials, percentage of error, and percentage of retrieval reported for addition trials as a function of group and problem size. Table 4 presents the corresponding ANOVAs.

Response times. As is typically found, large addition problems took longer to solve than small problems $(1,171 \mathrm{msec}$ vs. $927 \mathrm{msec})$. More important, those participants who solved multiplication problems first presented longer addition RTs on average (M-A, 1,118 msec) than did those participants who solved division problems first

Table 2

\begin{tabular}{|c|c|c|c|c|c|c|c|c|c|}
\hline \multirow[b]{2}{*}{ Source } & \multicolumn{3}{|c|}{ RT } & \multicolumn{3}{|c|}{$\%$ Errors } & \multicolumn{3}{|c|}{$\%$ Retrieval } \\
\hline & $F$ & $d f$ & $M S_{\mathrm{e}}$ & $F$ & $d f$ & $M S_{\mathrm{e}}$ & $F$ & $d f$ & $M S_{\mathrm{e}}$ \\
\hline \multicolumn{10}{|c|}{ Between Subjects } \\
\hline roup & 0.87 & 1,98 & $137,380.08$ & 2.89 & 1,98 & 20.67 & 0.71 & 1,48 & 467.30 \\
\hline \multicolumn{10}{|c|}{ Within Subjects: Size } \\
\hline ze & $124.76^{*}$ & 1,98 & $45,344.38$ & $200.93^{*}$ & 1,98 & 16.98 & $37.97^{*}$ & 1,48 & 45.28 \\
\hline roup $\times$ Size & 1.81 & 1,98 & $45,344.38$ & 0.87 & 1,98 & 16.98 & 0.62 & 1,48 & 45.28 \\
\hline
\end{tabular}

Note-RT, mean correct response time; \%Errors, mean percentage of incorrect responses; $\%$ Retrieval, mean reported use of direct retrieval. Fifty of the 100 participants provided strategy reports during context trials. See Table 1 for the corresponding means. ${ }^{*} p<.001$. 
Table 3

Mean Correct Response Times (RTs; in Milliseconds), Percentage of Errors, and Percentage of Retrieval Reported for Addition Trials by Group and Problem Size

\begin{tabular}{|c|c|c|c|c|c|}
\hline Condition & RT & $\% \mathrm{E}=$ & $\% \mathrm{OP}+$ & $\%$ Other & $\%$ Retrieval \\
\hline \multicolumn{6}{|c|}{ Small Additions } \\
\hline M-A group & 992 & 5.7 & 5.1 & 0.6 & 77.3 \\
\hline D-A group & 861 & 2.1 & 1.8 & 0.3 & 92.9 \\
\hline$M-D$ & +131 & +3.6 & +3.3 & +0.3 & -15.6 \\
\hline \multicolumn{6}{|c|}{ Large Additions } \\
\hline M-A group & 1,243 & 4.8 & 0.3 & 4.5 & 57.9 \\
\hline D-A group & 1,099 & 3.3 & 0.1 & 3.2 & 66.6 \\
\hline$M-D$ & +144 & +1.5 & +0.2 & +1.3 & -8.7 \\
\hline
\end{tabular}

Note- $\% \mathrm{E}=\% \mathrm{OP}+\% \mathrm{Other} \% \mathrm{OP}=$ mean percentage of trials involving operation errors (e.g., $2+3=" 6 ", 3+4=" 12 ")$. \%Other = mean percentage of trials involving other (i.e., nonoperation) errors, Small, problems with both addends $\leq 5(n=20)$; large, at least one addend $>5(n=52)$.

(D-A, $980 \mathrm{msec}$ ). This is consistent with our expectation that the multiplication context would interfere with addition performance.

Errors. The participants also made more errors of addition following multiplication ( $\mathrm{M}-\mathrm{A}, 5.3 \%)$ than following division ( $\mathrm{D}-\mathrm{A}, 2.7 \%$ ), and as was predicted, this effect was more marked for small problems $(+3.6 \%$ for small problems, as compared with $+1.5 \%$ for large problems). This group $\times$ size effect was due entirely to operation errors (i.e., multiplication confusions) such as 2 $+3=6$ or $3+4=12$. There were no addition errors corresponding to division confusions (e.g., $8+2=4$ ). Table 3 includes the mean percentage of trials involving operation errors versus nonoperation errors. Eighty-nine percent of operation errors occurred on the small problems, whereas only $4 \%$ of nonoperation errors involved the small problems (see Campbell, 1994). As Table 3 shows, relative to $\mathrm{D}-\mathrm{A}$, the $\mathrm{M}-\mathrm{A}$ condition increased operation errors more on small addition problems $(+3.3 \%)$ than on large problems $(+0.2 \%)$. The error data thereby indicate that multiplication-related interference was greater for small than for large addition problems.

Reported use of retrieval. M-A participants reported using retrieval less often on addition problems than did D-A participants $(63.3 \%$ vs. $73.9 \%)$. As in previous research, the participants used retrieval more frequently on small than on large problems ( $85.1 \%$ vs. $62.3 \%$ ). Importantly, however, group and problem size interacted. Those participants who solved multiplication first showed a smaller effect of problem size on retrieval use (77.3\% vs. $57.9 \%$ for small and large problems, respectively) than did those participants who solved division first $(92.9 \%$ vs. $66.6 \%$ ). The pattern of means indicates that the multiplication context reduced retrieval use more for small addition problems $(-15.6 \%)$ than for large problems $(-8.7 \%)$. Thus, as was expected from the DOA model, greater retrieval interference for small problems was associated with a greater increase in nonretrieval strategies. This effect was due mainly to greater reported use of counting by $\mathrm{M}-\mathrm{A}$ participants than by $\mathrm{D}-\mathrm{A}$ participants, especially for smaller problems: Relative to $\mathrm{D}-\mathrm{A}, \mathrm{M}-\mathrm{A}$ participants reported counting 3.4 times more often for small additions ( $20.0 \%$ vs. $5.9 \%$ ), but only 1.4 times more often for large additions ( $24.7 \%$ vs. $17.7 \%$ ). The groups reported similar rates of transformation for addition (M-A: small, 2.5\%; large, 17.2\%; D-A: small, 1.2\%; large, $14.8 \%)^{4}$

We also examined another prediction regarding strategy choice that follows from the DOA model: Practicing a number fact should increase the relative strength of the correct association and thereby increase the probability of retrieval. Thus, given evidence that identical-element number facts (e.g., $2 \times 6$ and $6 \times 2,5+7$ and $7+5$ ) access the same memory node (Rickard \& Bourne, 1996), there should be more retrieval reported in the second block of addition trials. Consistent with this, reported retrieval for addition increased from the first $(M=66.2 \%)$ to the second $(M=71.0 \%)$ block $[t(99)=-5.22, S E=$ $0.92, p<.001]$.

Addition performance on reported retrieval and nonretrieval trials. Group $\times$ size $\times$ strategy analyses of addition performance were restricted by the relatively low rate of nonretrieval reported: $49 \%$ of the participants contributed no observations to at least one size $\times$ strategy cell..$^{5}$ A strategy (retrieval vs. nonretrieval) $\times$ size ANOVA (i.e., collapsing over group; $n=51$ ) indicated faster RTs for retrieval (small problems, $930 \mathrm{msec}$; large, $1,041 \mathrm{msec}$ ) than for nonretrieval [small, $1,179 \mathrm{msec}$; large, $1,479 \mathrm{msec} ; F(1,50)=95.13, M S_{\mathrm{e}}=63,179.83, p<$ $.001]$. The problem size effect was much greater for non-

Table 4

Group $\times$ Size Analyses of Variance of Response Times, Percentage of Errors, and Percentage of Retrieval Reported for Addition Trials

\begin{tabular}{|c|c|c|c|c|c|c|c|c|c|}
\hline \multirow[b]{2}{*}{ Source } & \multicolumn{3}{|c|}{ RT } & \multicolumn{3}{|c|}{$\%$ Errors } & \multicolumn{3}{|c|}{$\%$ Retrieval } \\
\hline & $F$ & $d f$ & $M S_{\mathrm{e}}$ & $F$ & $d f$ & $M S_{\mathrm{e}}$ & $F$ & $d f$ & $M S_{\mathrm{c}}$ \\
\hline \multicolumn{10}{|c|}{ Between Subjects } \\
\hline Group & $8.35 \dagger$ & 1,98 & $113,472.21$ & $10.53+$ & 1,98 & 31.35 & $8.22 \dagger$ & 1,98 & 897.86 \\
\hline \multicolumn{10}{|c|}{ Within Subjects: Size } \\
\hline Size & $141.29 \ddagger$ & 1,98 & $21,161.74$ & 0.08 & 1,98 & 12.16 & $187.75^{+}$ & 1,98 & 139.28 \\
\hline Group $\times$ Size & $0.10^{\circ}$ & 1,98 & $21,161.74$ & $4.37^{*}$ & 1,98 & 12.16 & $4.28^{*}$ & 1,98 & 139.28 \\
\hline
\end{tabular}


retrieval (+300 msec) than for retrieval $[+111 \mathrm{msec}$; $\left.F(1,50)=22.19, M S_{\mathrm{e}}=20,474.43, p<.001\right]$. As the corresponding analysis of errors indicated only a main effect of strategy, we compared mean error rates for retrieval versus nonretrieval, collapsing over both group and size. The overall error rate for retrieval $(M=2.8 \%, S D=3.9)$ was much lower than that for nonretrieval $[M=8.1 \%$, $S D=13.8 ; t(93)=3.62, S E=1.466, p<.001]$. Nonetheless, most operation errors $(76 \%)$ were classified by the participants as retrieval trials. The data are consistent with previous research indicating better performance for retrieval than for nonretrieval procedures for simple addition (LeFevre, Sadesky, \& Bisanz, 1996).

\section{DISCUSSION}

Addition following multiplication was $14 \%$ slower and produced twice as many errors as addition following division. These effects cannot be attributed to different performance sets established during context trials: Speed, accuracy, and reported use of retrieval versus nonretrieval were quite similar for the multiplication and the division context trials. Furthermore, selective promotion of operation errors on small addition problems (e.g., $2+$ $5=10$ ) by the multiplication context provides direct evidence that multiplication, per se, interfered with addition performance.

The interference we observed may be related to retrieval-induced forgetting that is observed in episodic memory tasks. M. C. Anderson, Bjork, and Bjork (1994; M. C. Anderson \& McCulloch, 1999) found that repeated, cued-retrieval practice of a subset of previously studied items interfered with cued retrieval of semantically related unpracticed items. Furthermore, M. C. Anderson et al. found that such retrieval-induced forgetting was greater for strong category exemplars than for weak exemplars. This finding parallels our results in that smallnumber additions, which have strong memory representations, were more susceptible to interference from previous multiplication than were the weaker, large-number additions. Selective interference with small additions may occur because the corresponding small multiplications $(2 \times 5=10)$ have stronger memory representations than do large multiplication problems $(7 \times 8=56)$. Consequently, small addition problems may be more likely to activate and encounter interference from their multiplication counterparts. Alternatively, the multiplication practice phase may entail a suppression of addition facts that persists into the addition phase (e.g., $2+5=7$ may be inhibited during retrieval of $2 \times 5=10$ ). If such suppression is proportional to the strength of the competing item, we would expect more suppression in connection with the relatively strong, small-number additions (see M. C. Anderson et al., 1994). Thus, the decrease in the relative strength of the correct addition fact could reflect increased strength of the competing multiplication fact, suppression of the addition fact, or both.
Several of our findings support the DOA model of arithmetic strategy choice (Siegler, 1988). First, nonretrieval strategies for addition were reported $40 \%$ more often by M-A participants $(1,322$ trials) than by $\mathrm{D}-\mathrm{A}$ participants (940 trials). This effect is predicted by the DOA model because it assumes that procedural strategies are used when the strength of the correct answer is relatively low. Consequently, manipulations that reduce the relative strength of the correct fact, such as practicing associative competitors, ought to increase procedure use, as was observed. Second, the analysis of operation errors indicated that small addition problems encountered more interference from multiplication than did large problems. As was expected from the DOA model, the multiplication context produced a corresponding greater increase, relative to division, in reported nonretrieval for small problems than for large problems. ${ }^{6}$ Finally, the DOA model predicts that strengthening the correct fact should reduce interference and increase the probability of retrieval use. Consistent with this, reported retrieval increased in the second block of addition. Our findings therefore confirm predictions of the DOA model regarding both manipulations that decrease use of retrieval (i.e., strengthening of competing associations) and manipulations that increase use of retrieval strategies (i.e., strengthening of the correct association).

Although the strategy reports must be interpreted cautiously (see Kirk \& Ashcraft, 1997), the pattern of addition strategy reports and performance data was coherent and consistent with previous research. Reported retrieval for addition by D-A participants (73\%) matched previous research testing North American university students' simple addition $(73 \%$ in Geary, $1996 ; 71 \%$ in LeFevre, Sadesky, \& Bisanz, 1996). Also consistent with expectations, it was observed that although retrieval was the predominant strategy reported, nonretrieval strategies were reported more often for large than for small problems. This is expected, because small problems are encountered more frequently and may generally be easier to memorize (Geary, 1996). Moreover, RTs were substantially longer for nonretrieval than for retrieval trials, and this effect was greater for large problems. The strategy $\times$ size RT interaction would arise because nonretrieval procedures generally require more (or more difficult) steps for large than for small problems. Nonretrieval strategies were also about twice as error prone, as compared with retrieval, an effect that would arise from having to perform multistep procedures under relatively high speed pressure. Other features of the data further reinforce the validity of the strategy reports. For example, the increase in reported nonretrieval by the M-A group was due mainly to a greater use of counting strategies, especially for small problems. Counting is a more effective strategy for small than for large problems, because the correct solution can be obtained with a smaller number of increments. Similarly, counting is a more straightforward procedure for addition than for either multiplication 
or division (i.e., counting for the latter entails repeated addition or repeated subtraction). Consequently, as was observed, we would expect higher rates of counting reported for addition ( $18.9 \%$ overall) than for division and multiplication ( $7.1 \%$ overall).

The strategy results fit well with theory and are consistent with the performance data, but they should not be generalized to broadly characterize North American adults. Our context manipulation demonstrates that strategy reports (and presumably strategy use) depend critically on the testing conditions. For example, the common experimental practice of testing large numbers of closely related problems one after the other in rapid succession undoubtedly amplifies retrieval interference (Campbell \& Arbuthnott, 1996) and, based on the present findings, would tend to inflate procedure use. Furthermore, there are striking inconsistencies among studies in the observed rates of retrieval versus nonretrieval strategies. For simple multiplication, LeFevre, Bisanz, et al. (1996) found about $85 \%$ reported retrieval by adults, whereas LeFevre and Morris (1999) found only 59\%. The latter is also much lower than the $87 \%$ retrieval for simple multiplication found here. Similarly, LeFevre and Morris found $45 \%$ reported retrieval for Canadian undergraduates' simple division, which again is much lower than the $90 \%$ found here. These discrepancies may reflect population or sampling differences, different speed-accuracy criteria, experimental design, strategy instructions, and so on. Indeed, adults' strategy reports for simple arithmetic can be dramatically biased by instructions that differentially characterize the normative use of retrieval versus nonretrieval strategies (Kirk \& Ashcraft, 1997). All of these considerations recommend caution in generalizing the strategy results from individual experiments.

We formulated our predictions on the basis of the relatively simple DOA model (Siegler, 1988), but a complete model of adults' strategy choices may require the more complex assumptions of the ASCM (Siegler \& Shipley, 1995). In contrast to the DOA model, retrieval is not necessarily the default strategy in the ASCM. Instead, a strategy is selected on the basis of knowledge of its efficiency (i.e., speed and probability of success), which is implemented at three levels: the global level (the level at which the overall efficiency of a strategy is represented), the featural level (i.e., the level at which a subclass of problems may be strongly associated with a specific strategy), and the local level (i.e., the level at which specific problems may be directly associated with a specific strategy). Such featural or local influences may determine adults' choices among nonretrieval strategies for simple arithmetic (e.g., counting vs. transformation). Furthermore, some adults may know that their retrieval skill is not reliable for large-number problems and, therefore, may use a procedure as the default strategy for these cases. This potentially would contribute to our finding that strategy selection for large problems was less sensitive to retrieval interference than was that for small problems.

\section{CONCLUSIONS}

In summary, our findings demonstrate that adults' correct RT, errors, and reported use of nonretrieval procedures for simple addition are substantially increased in the high-interference context created by previously performing simple multiplication. These effects are all predicted by Siegler's (1988) DOA model of retrieval and strategy choice for simple arithmetic. The performance data, specific strategies reported, and group $\times$ size interaction on reported retrieval reinforce the conclusion that interference affected strategy choice. The results also support theories that identify a central role for associative interference in cognitive arithmetic (e.g., Campbell, 1995; Siegler \& Shrager, 1984; Zbrodoff, 1995).

\section{REFERENCES}

Anderson, J. R., \& Lebiere, C. (1998). The atomic components of thought. Hillsdale, NJ: Erlbaum.

ANderson, M. C., Bjork, R. A., \& Bjork, E. L. (1994). Remembering can cause forgetting: Retrieval dynamics in long-term memory. Journal of Experimental Psychology: Learning, Memory, \& Cognition, 20, 1063-1087.

ANDERSON, M. C., \& MCCulloch, K. C. (1999). Integration as a general boundary condition on retrieval-induced forgetting. Journal of Experimental Psychology: Learning, Memory, \& Cognition, 25, 608629.

AshCRAFT, M. H. (1992), Cognitive arithmetic: A review of data and theory. Cognition, 44, 75-106.

AshCRAFT, M. H., \& ChristY, K. S. (1995). The frequency of arithmetic facts in elementary texts: Addition and multiplication in grades $1-6$. Journal for Research in Mathematics Education, 5, 396-421.

Campbell, J. I. D. (1994). Architectures for numerical cognition. Cognition, 53, 1-44.

CAMPBELl, J. I. D. (1995). Mechanisms of simple addition and multiplication: A modified network-interference theory and simulation. Mathematical Cognition, 1, 121-164.

Campbell, J. I. D. (1997). On the relation between skilled performance of simple division and multiplication. Journal of Experimental Psychology: Learning, Memory, \& Cognition, 23, 1140-1159.

Campbell, J. I. D. (1999). Division by multiplication. Memory \& Cognition, 27, 791-802.

CAMPBELl, J. I. D., \& ARButhnOTT, K. D. (1996). Inhibitory processes in sequential retrieval: Evidence from variable-lag repetition priming. Brain \& Cognition, 30, 59-80

GEARY, D. C. (1996). The problem-size effect in mental addition: Developmental and cross-national trends. Mathematical Cognition, 2, 63-93.

Geary, D. C., Frensch, P. A., \& Wiley, J. D. (1993). Simple and complex mental subtraction: Strategy choice and speed-of-processing differences in younger and older adults. Psychology \& Aging, 8, 242256.

GEARY, D. C., \& WILEY, J. G. (1991). Cognitive addition: Strategy choice and speed-of-processing differences in young and elderly adults. $P s v$ chology \& Aging, 6, 474-483.

Kirk, E. P., \& AshCrafT, M. H. (1997, November). Verbal reports on simple addition: A demanding task. Poster presented at the 38th Annual Meeting of the Psychonomic Society, Philadelphia.

LeFevre, J., Bisanz, J., Daley, K. E., Buffone, L., Greenham, S. L., \& SADESKY, G. S. (1996). Multiple routes to solution of single-digit multiplication problems. Journal of Experimental Psychologv: General, 125, 284-306.

LEFEVRE, J., \& LIU, J. (1997). The role of experience in numerical skill: Multiplication performance in adults from China and Canada. Mathematical Cognition, 3, 31-62. 
LeFevre, J., \& Morris, J. (1999). More on the relation between division and multiplication in simple arithmetic: Evidence for mediation of division solutions via multiplication. Memory \& Cognition, 27, 803-812.

LeFevre, J., Sadesky, G. S., \& Bisanz, J. (1996). Selection of procedures in mental addition: Reassessing the problem size effect in adults. Journal of Experimental Psychology: Learning, Memory, \& Cognition, 22, 216-230.

LoGAN, G. D. (1988). Toward an instance based theory of automatization. Psychological Review, 95, 492-527.

LOGAN, G. D., \& KLAPP, S. T. (1991). Automatizing alphabet arithmetic: I. Is extended practice necessary to produce automaticity? Journal of Experimental Psychology: Learning, Memory, \& Cognition, 17, 179-195.

McCloskey, M., \& Lindemann, A. M. (1992). MATHNET: Preliminary results from a distributed model of arithmetic fact retrieval. In J. I. D. Campbell (Ed.), The nature and origins of mathematical skills (pp. 365-409). Amsterdam: Elsevier.

Miller, K. F., \& Paredes, D. R. (1990). Starting to add worse: Effects of learning to multiply on children's addition. Cognition, 37, 213242.

NoËL, M.-P., FIAS, W., \& BRYSBaERT, M. (1997). About the influence of the presentation format on arithmetical-fact retrieval processes. Cognition, 63, 335-374.

Rickard, T. C., \& Bourne, L. E., JR. (1996). Some tests of an identical elements model of basic arithmetic skills. Journal of Experimental Psychology: Learning, Memory, \& Cognition, 22, 1281-1295.

SIEGLER, R. S. (1988). Strategy choice procedures and the development of multiplication skills. Journal of Experimental Psychology: General, 106, 250-264.

Siegler, R. S., \& LemaiRe, P. (1997). Older and younger adults' strategy choices in multiplication: Testing predictions of ASCM using the choice/no-choice method. Journal of Experimental Psychology: General, 126, 71-92.

Siegler, R. S., \& Shipley, C. (1995). Variation, selection, and cognitive change. In G. Halford \& T. Simon (Eds.), Developing cognitive competence: New approaches to process modelling (pp. 31-76). Hillsdale, NJ: Erlbaum.

Siegler, R. S., \& Shrager, J. (1984). A model of strategy choice. In C. Sophian (Ed.), Origins of cognitive skills (pp. 229-293). Hillsdale, NJ: Erlbaum

SoHn, M.-O., \& Carlson, R. (1998). Procedural frameworks for simple arithmetic skills. Journal of Experimental Psychology: Learning. Memory, \& Cognifion, 24, 1052-1067.

ZBrodofF, N. J. (1995). Why is $9+7$ harder than $2+3$ ? Strength and interference as explanations of the problem-size effect. Memory \& Cognition, 23, 689-700.

Zbrodoff, N. J., \& Logan, G. D. (1986). On the autonomy of mental processes: A case study of arithmetic. Journal of Experimental Psychology: General, 115, 118-130.

\section{NOTES}

1. Current arithmetic models differ in assumptions about the format of number fact representations and the specific mechanisms of retrieval and interference, but these differences are not crucial here. The models generally agree that retrieval performance depends on the relative strength of the correct fact in the context of multiple related facts that are activated in parallel (e.g., J. R. Anderson \& Lebiere, 1998, Chap. 9; Campbell, 1995; McCloskey \& Lindemann, 1992; Seigler, 1988).
2. Those participants who provided strategies during the multiplication or division context trials produced marginally faster addition RTs $(M=1,054 \mathrm{msec}, S D=253)$, relative to those who did not $[M=$ $1,151 \mathrm{msec}, S D=304 ; t(98)=1.74, S E=55.91, p=.081]$. Apart from this, whether or not strategies were collected during context trials had no significant effects on RT, error rates, or reported use of retrieval, either on the context trials or on the subsequent addition trials. Consequently, we do not consider this manipulation as a factor in the following analyses.

3. It is worth noting that for the $89 \%$ of the context trials reported as retrieval (i.e., excluding the more variable nonretrieval trials), RTs were significantly faster for multiplication (small problems, $849 \mathrm{msec}$; large, $1,099 \mathrm{msec}$ ) than for division [small, $984 \mathrm{msec}$; large, $1,232 \mathrm{msec}$; $\left.F(1,48)=4.58, M S_{\mathrm{e}}=98,200.06, p=.037\right]$. Similarly, the retrieval error rate was lower for multiplication (small, $0.6 \%$; large, $6.6 \%$ ) than for division [small, $2.0 \%$; large, $8.7 \% ; F(1,48)=4.99, M S_{\mathrm{e}}=15.60, p=.030$ ].

4. Does the the greater difficulty of addition following multiplication than of that following division depend on the greater visual similarity of the addition and the multiplication stimuli, relative to the addition and the division stimuli? To pursue this, we conducted analyses that included only the addition items with visually similar multiplication and division stimuli (i.e., division problems with single-digit dividends). These addition stimuli (i.e., $4+2,6+3,6+2,8+2,8+4,9+3$ ) appear to be equivalent in visual similarity to the corresponding division $(4 \div 2,6 \div 3,6 \div 2,8 \div 2,8 \div 4,9 \div 3)$ and multiplication (i.e., $4 \times 2,6 \times 3,6 \times 2,8 \times 2,8 \times 4,9 \times 3$ ) items. Each addition item was presented in one operand order or the other (i.e., $2+4$ or $4+2$ ) in each of the two addition blocks, providing 12 trials per subject. We used $t$ tests to compare the groups on (1) percent retrieval [M-A, $66.0 \%$, vs. $\mathrm{D}-\mathrm{A}, 79.3 \% ; t(98)=-2.51, S E=5.30, p=.014 \mathrm{]},(2)$ percentage of errors: [M-A, $6.8 \%$, vs. D-A, $3.8 \% ; t(98)=2.30, S E=1.30, p=.023$ ], and (3) RT $[1,118 \mathrm{msec}$ vs. $1,023 \mathrm{msec} ; t(98)=1.79, S E=53.28, p=$ .077 ]. Thus, poorer addition after multiplication than after division did not depend on differences in the visual similarity of corresponding multiplication or division items in the context trials.

5 . We did examine group $\times$ size effects on addition RT and errors separately for retrieval and nonretrieval trials. The retrieval analyses reproduced the same pattern of statistical effects as the overall analysis. In contrast, there were no significant group-related differences in the nonretrieval analyses. The absence of significant group differences for nonretrieval trials must be interpreted cautiously, however. Relative to reported retrieval trials, the nonretrieval analyses were based on about half as many participants and on fewer observations per participant (i.e., because of the relatively low rate of nonretrieval reported) and involved much greater variances.

6. In theory, greater interference for small problems should also be observed in RTs, whereas the ANOVA indicated slower addition RTs overall for the M-A group, relative to the D-A group, but no interaction. RT effects owing to a greater increase in use of procedural strategies for small than for large problems may be offset by the fact that such strategies are generally faster for small problems. Furthermore, the strong group $x$ size interaction observed in the analysis of errors may indicate that interference was largely "absorbed" by errors, making the RT analysis relatively insensitive to a group $\times$ size effect.

(Manuscript received May 12, 1999; revision accepted for publication December 20, 1999.) 